DISEÑO DEL PERFIL DE INGRESO PARA LA INGENIERÍA EN GESTIÓN EMPRESARIAL DEL INSTITUTO SUPERIOR DE LIBRES.

\title{
Diseño del Perfil de Ingreso para la Ingeniería en Gestión Empresarial del Instituto Tecnológico Superior de Libres
}

\section{Design of the Entry Profile for Engineering in Business Management at the Instituto Tecnológico Superior de Libres}

\author{
Martínez Ángeles Dulce María*, Lobato Báez Mariana**, \\ Bello Ramírez Martha Irene***
}

\begin{abstract}
*Doctora en Estudios Sociales, Línea Economía. Instituto Tecnológico Superior de Libres. Email: dulcemaria.ma@itslibres.tecnm.mx, ORCID: https://orcid.org/0000-0003-0049-3397.

**Doctora en Planeación Estratégica y Dirección en Tecnologías. Instituto Tecnológico Superior de Libres. Email: mariana.lobato@upaep.edu.mx, ORCID: https://orcid.org/0000-0002-2607-2032.

***Maestra en Astrofísica. Instituto Tecnológico Superior de Libres. Email: martha.beram@gmail.com, ORCID: https://orcid.org/0000-0001-5949-3952.
\end{abstract}

Correo para recibir correspondencia: mariana.lobato@upaep.edu.mx 
DISEÑO DEL PERFIL DE INGRESO PARA LA INGENIERÍA EN GESTIÓN EMPRESARIAL DEL INSTITUTO SUPERIOR DE LIBRES.

\title{
RESUMEN
}

OBJETIVO: Diseñar un perfil de ingreso de la Ingeniería en Gestión Empresarial del Instituto Tecnológico Superior de Libres (ITSL), a partir de la experiencia derivada del proceso de acreditación del programa educativo por parte del Consejo se Acreditación de la Enseñanza de la Ingeniería A. C. (CACEI), pues se carecía de la claridad de los elementos que debía contener el perfil de ingreso dentro del sistema del Tecnológico Nacional de México (TECNM).

MATERIAL Y MÉTODO: Se realizó una revisión teórica conceptual del perfil de ingreso, así como de publicaciones aplicadas referente a perfiles de ingreso en el país. Se realizó una revisión de los perfiles de ingreso de todos los campus del TECNM que ofertan esta carrera en el país, con la finalidad de identificar puntos en común, que se concentran en tres dimensiones: Conocimientos, Habilidades y Actitudes. Por último, se aplica un instrumento de medición a la generación 2020 con una población de 45 alumnos para realizar inferencias sobre los elementos del perfil de ingreso.

RESULTADOS: Muestran que los alumnos reflejan las características del perfil "ideal" de ingreso diseñado, mostrando a la dimensión conocimientos como la más baja de las dimensiones analizadas con respecto al promedio obtenido.

CONCLUSIONES: Puede determinarse que es importante distinguir entre lo que es el perfil de ingreso de lo que es el perfil del estudiante, así como también que la elaboración de este perfil de ingreso puede proponerse de manera nacional ante el sistema del Tecnológico Nacional de México (TECNM), para todos los campus que ofertan la Ingeniería en Gestión Empresarial.

PALABRAS CLAVE: Perfil de ingreso. Perfil del estudiante. Dimensiones. TECNM. CACEI.

\begin{abstract}
OBJECTIVE: To design an entry profile for the Business Management Engineering at the Instituto Tecnológico Superior de Libres (ITSL by its initials in Spanish), based on the experience derived from the accreditation process of its educational program by the Engineering Teaching Accreditation Council, Civil Association (CACEl by its abbreviation in Spanish), since
\end{abstract}


DISEÑO DEL PERFIL DE INGRESO PARA LA INGENIERÍA EN GESTIÓN EMPRESARIAL DEL INSTITUTO SUPERIOR DE LIBRES.

there was a lack of clarity about the elements that the entry profile should contain within the system of the Tecnológico Nacional de Mexico (TECNM).

MATERIAL AND METHOD: It was carried out a conceptual theoretical review of the entry profile, as well as of publications regarding to entry profiles applied in the country. It was also conducted a review of the entry profiles of all TECNM campuses that offer this career in the country, to identify common points, which were concentrated in three dimensions: Knowledge, Skills and Attitudes. Finally, a measurement instrument was applied to the 2020 generation with a population of 45 students to make inferences about the elements of the entry profile.

RESULTS: They reveal that the students reflect the characteristics of the "ideal" entry profile designed, showing the knowledge dimension, as the lowest of the analyzed dimensions with respect to the average obtained.

CONCLUSIONS: It can be said that it is important to distinguish the difference between what the entry profile is, and what the student's profile is; furthermore, the elaboration of this entry profile can be proposed at national level to the system of the Tecnológico Nacional de Mexico (TECNM), for all campuses that offer Business Management Engineering.

KEY WORDS: Entry profile. Student profile. Dimensions. TECNM. CACEI.

\section{INTRODUCCIÓN}

Uno de los problemas más comunes que se presentan con los estudiantes en el nivel superior tiene que ver con la deserción escolar, o bien con los índices de reprobación. Generalmente esto se alude al papel de los profesores y los métodos de enseñanza aprendizaje, pero se omite por mucho la relevancia del perfil de ingreso de los estudiantes como probable explicación de esta situación. Esto se debe, en gran medida, al proceso de la masificación de la educación superior, que consistió en generar universidades para que la población pudiera acceder a un nivel formativo superior; en consecuencia, el incremento de población estudiantil ha sido muy evidente, pero no se traduce en el número de profesionistas que existen en el país. 
DISEÑO DEL PERFIL DE INGRESO PARA LA INGENIERÍA EN GESTIÓN EMPRESARIAL DEL INSTITUTO SUPERIOR DE LIBRES.

Si bien, ha sido importante que haya mayor acceso a la educación superior, estos problemas persisten porque no se ha puesto atención a los requerimientos necesarios de cada disciplina que los aspirantes deban cumplir. Hay más interés por llenar los espacios disponibles en las universidades que por verificar que los estudiantes que quieren ingresar a ciertas carreras universitarias, cumplan con los requisitos necesarios para concluir con éxito su proceso educativo de nivel superior.

Un primer elemento de interés es el promedio del antecedente escolar para ingresar al nivel superior (Preparatoria, Bachillerato o equivalente), pues de acuerdo con de Garay y Sánchez (2011), se ha identificado una estrecha relación con las condiciones de rendimiento y alumnos regulares, sobre todo en el primer año de estudios, que es considerado como el determinante para la permanencia o el abandono de la carrera. Por esa misma razón, en la perspectiva de González, Castro y Bañuelos (2011), es importante abordar los problemas relacionados con la trayectoria escolar considerando no solo el promedio escolar, sino condiciones relacionadas con su entorno familiar y social. Entonces parece ser que el análisis del perfil de ingreso se vuelve complejo, y aun más cuando de analiza para cada una de las disciplinas de formación académica, pues existen diferencias, por ejemplo, en el perfil de ingreso de estudiantes de medicina con relación a estudiantes ciencias sociales, o ingeniería, etcétera.

Esta investigación, se enfatiza en el caso de la Ingeniería en Gestión Empresarial en Tecnológico Superior de Libres (ITSL). Esta ingeniería surge en 2008, como una idea de reemplazar a la Licenciatura en Administración, pero formalmente se establece en el año 2009 en el Instituto Tecnológico de Matamoros (Guerra, Guzmán y Valdéz, 2016). Desde entonces, el programa educativo se expandió por todos los campus del TECNM en el país, llegando actualmente a 195 planteles (TECNM, 2020). En el caso del ITSL, se establece en el año 2012 y actualmente, se cuentan con cinco generaciones de egresados.

Este documento presenta una revisión de los estudios más relevantes sobre el análisis del perfil de ingreso en el país, así como también algunas definiciones del perfil de ingreso, que permitirá operacionalizar este concepto de manera empírica. Posteriormente, se presenta la metodología utilizada par definir el perfil de ingreso ideal de un estudiante que quiera ingresar a la carrera de Ingeniería en Gestión Empresarial en el ITSL y emitir una serie de comentarios finales que concluyan los hallazgos más importantes de esta investigación. 
DISEÑO DEL PERFIL DE INGRESO PARA LA INGENIERÍA EN GESTIÓN EMPRESARIAL DEL INSTITUTO SUPERIOR DE LIBRES.

\section{Sobre el análisis del perfil de ingreso}

Dentro de la revisión de la literatura, se pudo identificar que hay mayor interés por presentar investigaciones relacionadas con el perfil de egreso; o bien, con el tema de las trayectorias escolares. Esto se debe probablemente a que hay más énfasis en la importancia de la relación de la educación superior y su vínculo con el mercado laboral, y de ahí su relación con los perfiles de egreso, ya que estos reflejan el "producto final" del proceso educativo para pasar al proceso laboral. Esto llama mucho la atención, porque como mencionan González, Lara, Pineda y Crespo (2014), dentro del diseño curricular, el perfil de ingreso siempre está presente y se asume que los alumnos que lo cubren tendrán un desempeño y culminación de estudios exitoso. Por lo tanto, hay relación con la eficiencia terminal y los perfiles de los estudiantes inciden en su trayectoria académica (González, Castro y Bañuelos, 2011).

A grandes rasgos, se ubicaron diversos estudios donde más allá de analizar un perfil de ingreso, se analiza el perfil del estudiante que ingresa a nivel superior en términos socioeconómicos, demográficos y hábitos escolares. En el caso de la propuesta de Rojas (2014), se determinan un análisis comparativo de perfiles de alumnos que ingresan al área de Ciencias, Ciencias económicas y Ciencias sociales de la Universidad de Costa Rica, donde se identifica que el punto en común es el promedio escolar, además de mostrar un perfil socioeconómico de los estudiantes. En el caso de González, Castro y Bañuelos (2011), presentan el caso del área de Ciencias Químicas de la BUAP, en México, y determinan el perfil sociodemográfico de los estudiantes bajo la perspectiva de las trayectorias escolares. Garibotti y Ambroggio (2012), aplican un estudio para el caso de alumnos de Ciencias de la Educación, pero también presentan un perfil del estudiante en la Universidad Nacional de Córdoba en Argentina donde también se analizan características de los estudiantes en términos sociales y académicos. Lo mismo ocurre con el aporte de Vivas, Yañez y Pérez (2018), pero aplicado a estudiantes de primer ingreso de la Facultad de Ciencias Agrícolas de la Universidad Central de Ecuador; y de Esparza y López (2011), para el Escuela de Diseño de la Universidad de la Salle, Bajío, en México.

El trabajo de Alvarado (2007), resalta al realizar un contraste entre el perfil de ingreso ideal y el perfil de ingreso real de estudiantes de la Licenciatura en Gestión Turística en la Universidad Autónoma de Chiapas, pues considera adecuadamente una percepción conceptual para definir los perfiles ideales y reales, y así realizar su instrumento de medición. Es este caso, pone MARTÍNEZ-ÁNGELES D. M., LOBATO-BÁEZ M., BELLO-RAMÍREZ M. I. 
DISEÑO DEL PERFIL DE INGRESO PARA LA INGENIERÍA EN GESTIÓN EMPRESARIAL DEL INSTITUTO SUPERIOR DE LIBRES.

énfasis en aspectos educativos, no tanto en aspectos sociodemográficos, pero es una propuesta interesante, ya que refleja la realidad de que en México ninguna institución pide al pie de la letra el perfil de ingreso a los estudiantes que van a ingresar a una carrera universitaria, pero que las instituciones educativas deben trabajar en estrategias que les permitan a los estudiantes adquirir las características necesarias para cumplir el perfil de ingreso ideal. Al respecto, el trabajo de Ysunza y de la Mora (2007) identifica las prácticas académicas y culturales que los alumnos de nuevo ingreso en la UAM Xochimilco traían desde su formación en el nivel medio superior, y cómo estas favorecen o desfavorecen su incorporación al sistema universitario, señalando también que las universidades deben de considerar estos aspectos para cumplir con la tarea de su formación integral.

Dentro del análisis conceptual del término "perfil de ingreso" se hallaron algunas definiciones propuestas. García y López (2011), retoman el concepto de Perfil de ingreso de Chan (1999), y de la Universidad Politécnica de Madrid (2007) (también citado en Alvarado 2019), donde concluyen que:

El perfil de ingreso trata de una descripción de los conocimientos y otras destrezas necesarias que la persona debe poseer para la aceptación en un curso determinado; entonces un perfil de ingreso es un cúmulo de las características, conocimientos, habilidades y aptitudes necesarias para que un alumno ingrese a un curso ya que esto garantiza el término exitoso de los estudios que se inician (García y López, 2011, p. 5).

La investigación de González et al. (2014), también presenta definiciones de lo que es un perfil de ingreso, tomadas del Procedimiento de perfiles de ingreso de la Escuela Universitaria de Ciencia de la Salud de la Universidad de Jaén de España (2008), y de la Guía Operativa para la Elaboración, Presentación y Aprobación de Proyectos de Creación y Modificación de Planes y Programas de Estudios de Licenciatura de la UNAM (2006):

"Se alude al perfil de ingreso como la descripción conceptual de las características deseables en el alumno de nuevo ingreso en términos de conocimientos, habilidades y actitudes favorables para cursar y terminar con mayores posibilidades de éxito los estudios que inicia. Además, da cuenta de las opciones académicas cursadas, notas académicas obtenidas y datos de carácter sociológico de interés" [...] "aquel que expresa los conocimientos, habilidades y valores que idealmente, debe tener el aspirante antes de 
DISEÑO DEL PERFIL DE INGRESO PARA LA INGENIERÍA EN GESTIÓN EMPRESARIAL DEL INSTITUTO SUPERIOR DE LIBRES.

ingresar al nivel superior; características de las cuales, una buena parte debieron haberse desarrollado en el nivel anterior" (citado en González et al. 2014, p. 13).

Con base en las definiciones anteriores, señalan que en la evaluación de los programas educativos se pone énfasis en que los profesores y la infraestructura, son elementos suficientes para mejorar su calidad y la formación de los profesionistas, omitiendo así la importancia de perfil de ingreso. Afirman que no es suficiente evaluar las características educativas del alumno, sino que deben considerarse aspectos económicos, sociales y culturales, pues influyen significativamente en el desempeño escolar y están relacionados con las trayectorias escolares (deserción, permanencia y eficiencia terminal) (González, et al., 2014).

En resumen, se deduce que, en efecto, el perfil de ingreso de remite a las características (conocimientos, habilidades, actitudes) "ideales" que los alumnos que desean ingresar a una carrera deberían cubrir para incrementar las posibilidades de concluir con éxito su formación universitaria. De aquí, su relación con el tema de la trayectoria escolar que los trabajos anteriormente descritos han señalado.

En este trabajo, se pretende enfocar precisamente en las características ideales que un aspirante a cursar la ingeniería en Gestión Empresarial debe cubrir, independientemente del promedio y del análisis de su contexto. Por ello, no es menester reproducir la diversidad de aportes observados referentes a los perfiles de los estudiantes y no a analizar, como tal, un perfil de ingreso adecuado para la Ingeniería en Gestión Empresarial.

\section{MATERIAL Y MÉTODO}

Dentro del procedimiento para identificar el perfil "ideal" de ingreso, primeramente, se revisaron los perfiles de ingreso de los 195 planteles en el país donde se oferta la Ingeniería en Gestión Empresarial. Los elementos en común se ordenaron en la tabla siguiente, considerando las características que se ubicaron de manera conceptual en la revisión bibliográfica (Conocimientos, Habilidades, Actitudes). 
DISEÑO DEL PERFIL DE INGRESO PARA LA INGENIERÍA EN GESTIÓN EMPRESARIAL DEL INSTITUTO SUPERIOR DE LIBRES.

Tabla 1

Perfil "ideal" de ingreso de la Ingeniería en Gestión Empresarial

\begin{tabular}{lll}
\multicolumn{1}{c}{ Conocimientos } & \multicolumn{1}{c}{ Habilidades } & Actitudes \\
\hline Matemáticas & Observación & Emprendedurismo \\
Inglés básico & Análisis & Innovación \\
Administración y contabilidad básica & Síntesis & Liderazgo \\
Manejo de TIC's & Trabajo en equipo & Gestión \\
& Expresión oral y escrita & Reflexión y pensamiento crítico \\
& Comprensión lectora & Interés por temas económicos nacionales e \\
& & internacionales \\
& Creatividad & Responsabilidad
\end{tabular}

Fuente: Elaboración propia.

Una vez identificado este perfil, se diseñó un instrumento para ser aplicado a los alumnos de la carrera de Ingeniería en Gestión Empresarial del ITSL, con una población de 45 alumnos. Posteriormente de encuestó a la población de estudio para recolectar información respecto al perfil de ingreso "ideal" y verificar si los estudiantes se aproximan a él o no. El instrumento obtuvo un Alfa de Cronbach de .949, lo cual indica un alto nivel de fiabilidad, además de que se excluyó un caso, que representó el $2 \%$.

\section{RESULTADOS}

Dentro de los resultados obtenidos del instrumento aplicado, se obtiene en primer lugar un perfil sociodemográfico, que refleja que $65 \%$ de los estudiantes son mujeres y $35 \%$ son hombres, lo que refleja una tendencia nacional de la incorporación de las mujeres al nivel educativo superior. Con respecto a las edades, se observó que van desde los 18 hasta los 24 años, lo cual remite al hecho de que la incorporación de los estudiantes a la universidad atrae a jóvenes que han postergado el inicio de sus estudios universitarios, aunque la media es de 19 años y $60 \%$ de los encuestados tiene 18 años, que es la edad promedio para comenzar la universidad. El $94 \%$ son solteros, y el $80 \%$ no tiene hijos. $80 \%$ de los estudiantes tienen como antecedente escolar el bachillerato y el resto proviene de una formación en preparatoria, este aspecto parece ser relevante debido a que la formación en ciertos sistemas educativos de nivel medio superior, determinan sus aspiraciones para el nivel superior. 
DISEÑO DEL PERFIL DE INGRESO PARA LA INGENIERÍA EN GESTIÓN EMPRESARIAL DEL INSTITUTO SUPERIOR DE LIBRES.

La mayoría de los estudiantes de nuevo ingreso procede del municipio de Libres, justamente donde pertenece la institución, no obstante, representa el $23 \%$ de todos los alumnos de ingreso (Figura 1). Le siguen municipios que no solo pertenecen al estado de Puebla, sino también al estado de Tlaxcala, lo cual refleja la importancia que ha tenido esta carrera dentro de una zona geográfica determinada debido la influencia de diversas actividades económicas, sobre todo por el establecimiento de unidades económicas que demandan servicios relacionados con el área que ofrece la Ingeniería en Gestión Empresarial (Granjas Caroll de México en el municipio de Oriental, Puebla; Corpus Tráilers en el municipio de Cuapiaxtla, Tlaxcala, Driscoll's S. A., en Huamantla, Tlaxcala y en Cuyoaco, Puebla, así como las empresas Tamariz y Empacadora San Marcos en la zona de Rafael Lara Grajales, Puebla, por mencionar algunos).

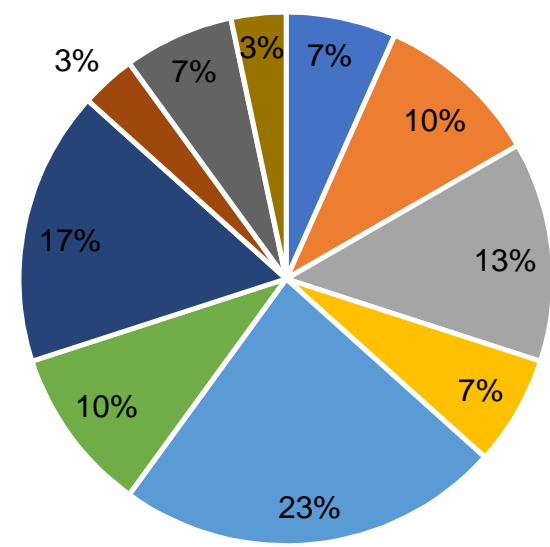

Figura 1. Municipios de origen de los estudiantes. Fuente: Elaboración propia.
- Altzayanca, Tlaxcala

- Cuyoaco, Puebla

- El Carmen Tequexquitla,

Tlaxcala

- Huamantla, Tlaxcala

- Libres, Puebla

- Nopalucan, Puebla

- Oriental, Puebla

- Puebla, Puebla

- Rafael Lara Grajales

- Zacapoaxtla, Puebla

Un hallazgo interesante es, que $80 \%$ de los estudiantes consideraron a la Ingeniería en Gestión Empresarial como su primera opción para cursar la carrera; los motivos por los cuales la eligieron se resumen en la Figura 2. 
DISEÑO DEL PERFIL DE INGRESO PARA LA INGENIERÍA EN GESTIÓN EMPRESARIAL DEL INSTITUTO SUPERIOR DE LIBRES.

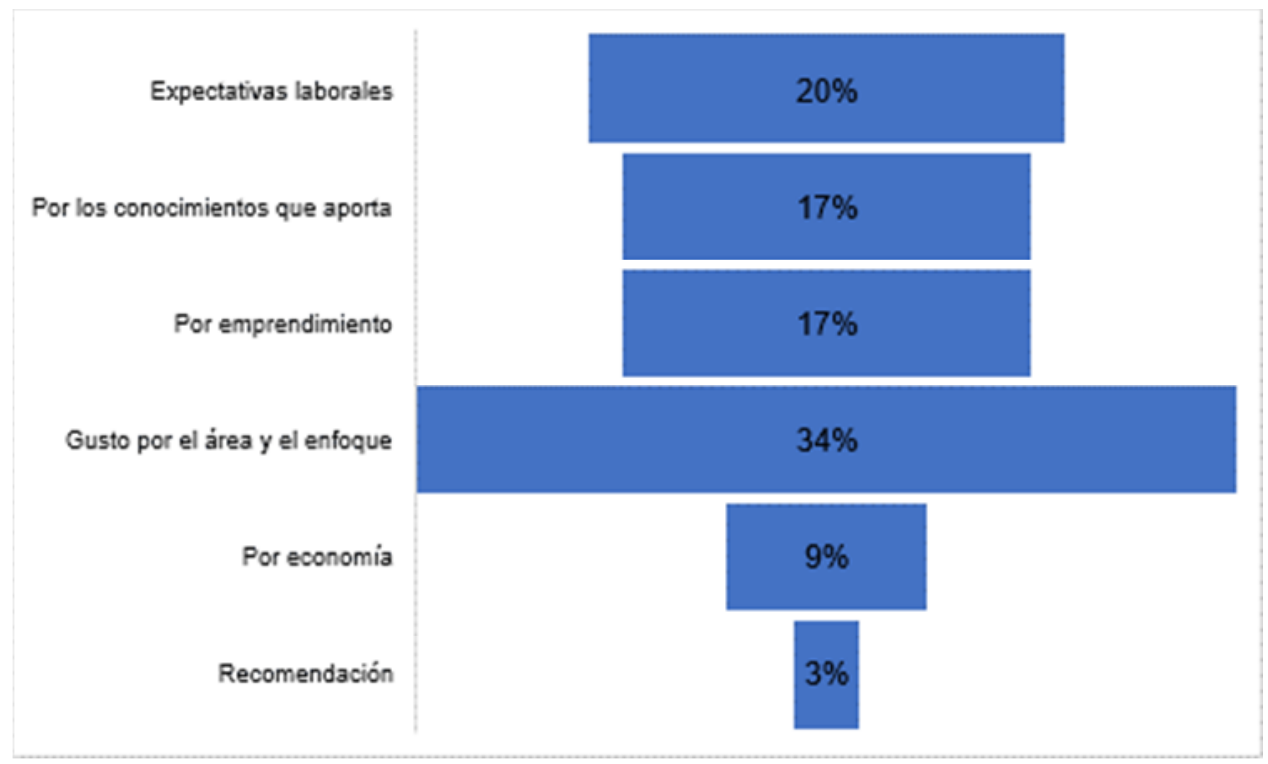

Figura 2. Motivos para estudiar la Ingeniería en Gestión Empresarial en el ITSL. Fuente: Elaboración propia.

Puede observarse, que el motivo principal se debe a que hay un interés por el área y el enfoque que se maneja en la ingeniería, pues como se señaló en el apartado de la introducción, esta carrera es novedosa al manejar un enfoque multidisciplinario, que contribuye a la solución de problemas que pueden surgir en una empresa, pero con una perspectiva integral. Asimismo, también influyen las expectativas laborales, pues se considera que cursar esta carrera incidirá positivamente en su inserción laboral, aunque también se considera la perspectiva del emprendimiento, lo cual es parte importante dentro del perfil de ingreso identificado.

Con respecto al contexto familiar de los estudiantes, en la Figura 3 se muestra el nivel educativo de los padres, donde puede observarse que la mayoría logró concluir estudios de nivel Secundaria. En algunos casos, cabe mencionar que las madres cuentan con mayor nivel educativo que los padres. 
DISEÑO DEL PERFIL DE INGRESO PARA LA INGENIERÍA EN GESTIÓN EMPRESARIAL DEL INSTITUTO SUPERIOR DE LIBRES.

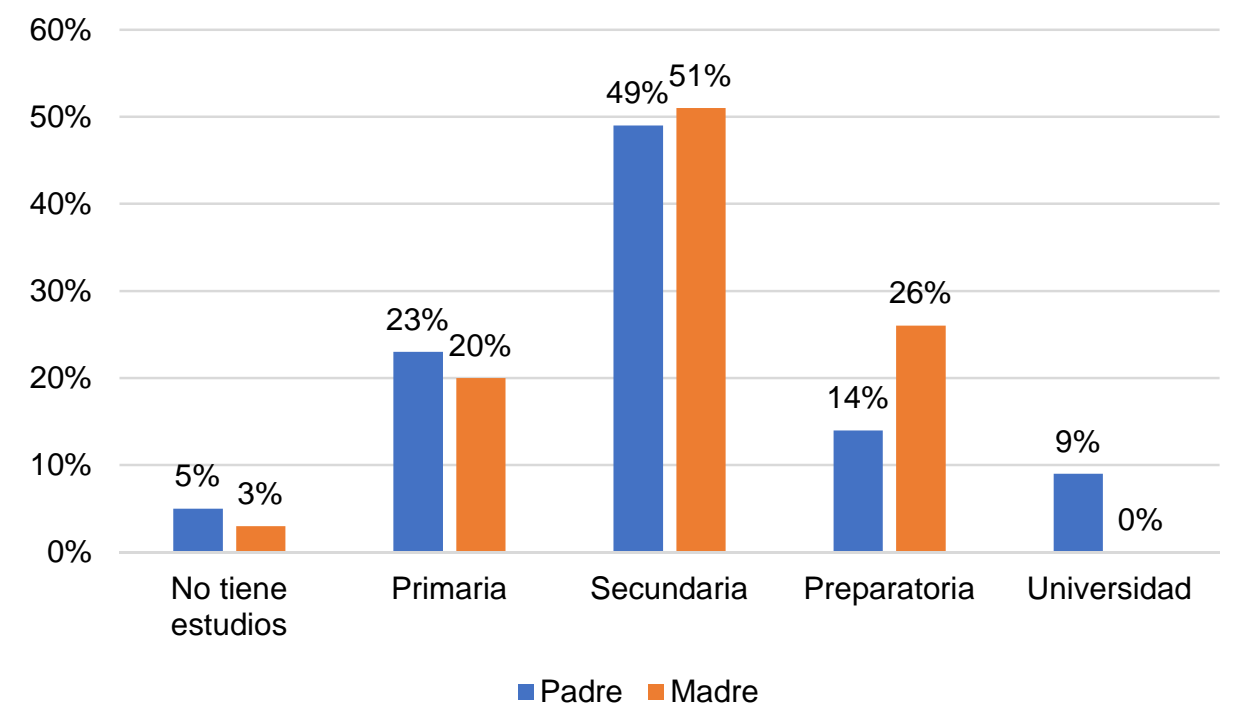

Figura 3. Nivel educativo de padres de los estudiantes de nuevo ingreso. Fuente: Elaboración propia.

En lo que se refiere a las ocupaciones laborales de los padres, se identificó que la mayoría se encuentra laborando como obreros, campesinos o como comerciantes, actividades propias de la región (Figura 4). Con respecto a las madres de familia, el $77 \%$ de ellas se dedica a labores del hogar (Figura 5). Estas situaciones, indican que la mayoría de los estudiantes se encuentra en un hogar donde solo se cuenta con un ingreso para cubrir todas sus necesidades.

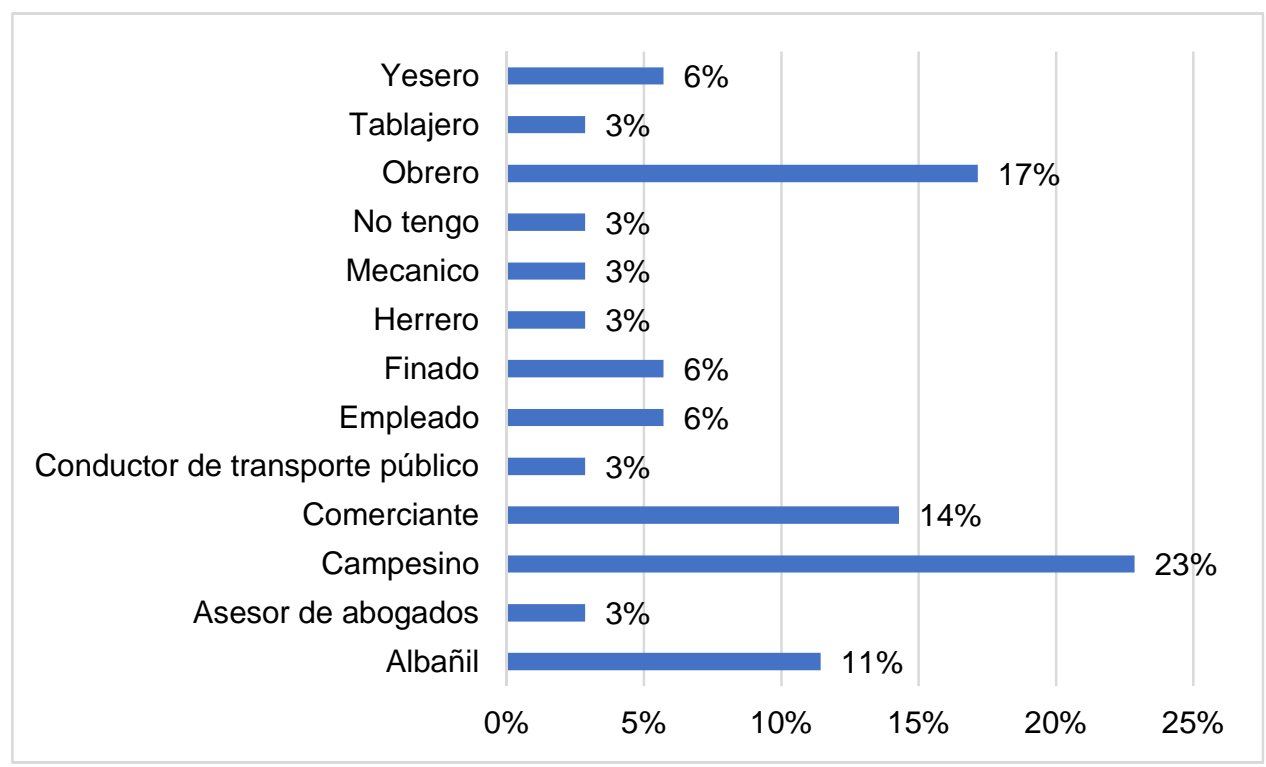

Figura 4. Ocupación de los padres de familia.

Fuente: Elaboración propia.

MARTÍNEZ-ÁNGELES D. M., LOBATO-BÁEZ M., BELLO-RAMÍREZ M. I.

HITOS DE CIENCIAS ECONÓMICO ADMINISTRATIVAS SEPTIEMBRE-DICIEMBRE 2021. Año 27, No. 79. Págs. $328-344$ 
DISEÑO DEL PERFIL DE INGRESO PARA LA INGENIERÍA EN GESTIÓN EMPRESARIAL DEL INSTITUTO SUPERIOR DE LIBRES.

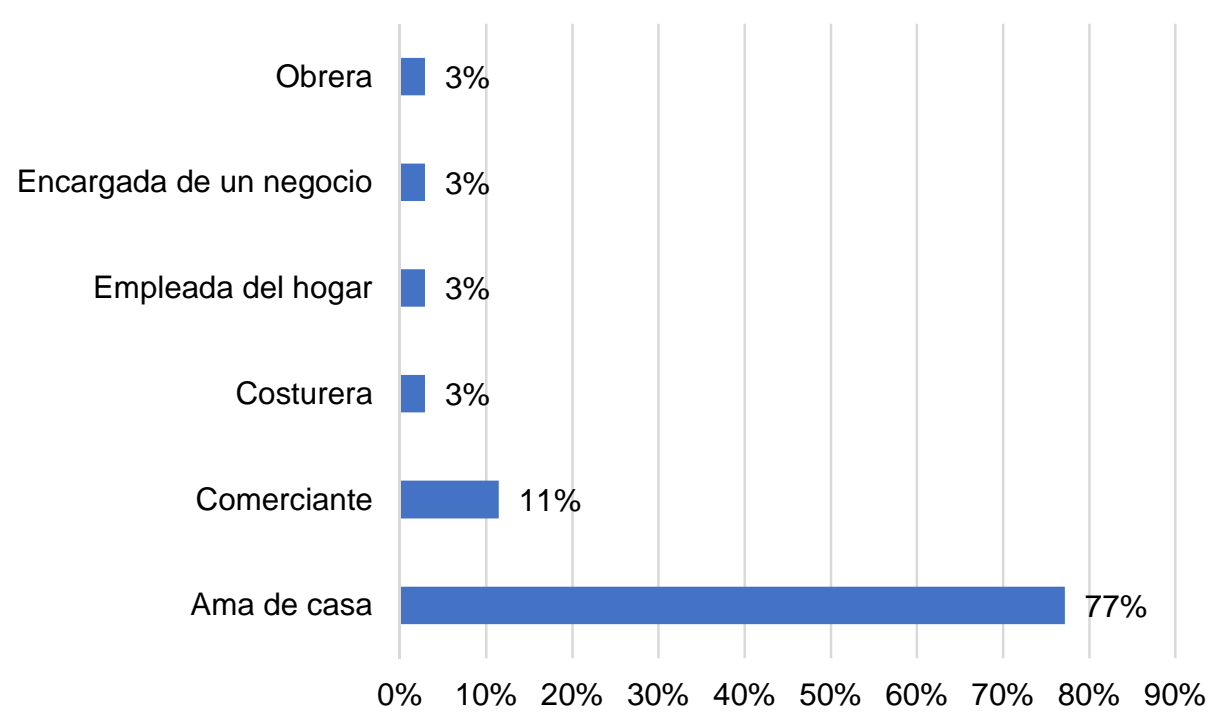

Figura 5. Ocupación de las madres de familia.

Fuente: Elaboración propia.

Lo anterior, influye en el resultado obtenido con respecto al financiamiento de los estudios de los alumnos de nuevo ingreso, ya que el $57 \%$ de los encuestados manifestó que sus padres lo cubren, mientras que el $43 \%$ se autofinancia, por lo cual trabaja y estudia al mismo tiempo. Por último, se halló que $40 \%$ de los estudiantes tiene acceso a un equipo de cómputo para realizar sus actividades escolares, mientras que el $57 \%$ tiene acceso a internet.

\section{Características del perfil de ingreso}

Se ha revisado que un aspecto relevante para el perfil de ingreso es el promedio escolar (de Garay y Sánchez, 2011). En el caso de este análisis, el promedio mínimo fue de 7 y el máximo de 9.30, y se obtuvo como valor de la media 7.9 de promedio escolar de los estudiantes de nuevo ingreso de la generación 2020 de la Ingeniería en Gestión Empresarial. Es un promedio aceptable, considerando que dentro del sistema del TECNM, se admiten a estudiantes que tengan promedio a partir de 6 , además de que la desviación estándar obtenida para esta variable fue de .74 .

En lo que se refiere a hábitos escolares se identificó, en promedio, que los estudiantes dedican 2.66 horas de estudio fuera de las clases; el valor mínimo fue de 1 hora y el valor máximo de 5 horas. Como se ha observado en la revisión de la literatura, este aspecto también es importante 
DISEÑO DEL PERFIL DE INGRESO PARA LA INGENIERÍA EN GESTIÓN EMPRESARIAL DEL INSTITUTO SUPERIOR DE LIBRES.

para determinar la probabilidad de que los alumnos puedan concluir exitosamente su formación universitaria, pues está relacionado con los indicadores de la trayectoria escolar.

En la siguiente tabla, se presenta el resumen de los valores obtenidos respecto a la valoración que los estudiantes de nuevo ingreso de la Ingeniería en Gestión Empresarial del ITSL proporcionaron respecto al perfil "ideal" de ingreso propuesto, pidiendo una autovaloración de las dimensiones de estudio de 1 a 10.

Tabla 2

Medición del perfil de ingreso

\begin{tabular}{|c|c|c|c|c|c|}
\hline Dimensión & Característica & Mínimo & Máximo & Media & Desviación Estándar \\
\hline \multirow{4}{*}{ Conocimientos } & Conocimientos matemáticos & 4 & 9 & 7.23 & 1.416 \\
\hline & Inglés básico & 1 & 8 & 5.09 & 1.837 \\
\hline & Conocimientos sobre administración y contabilidad básica & 2 & 10 & 6.49 & 2.147 \\
\hline & Manejo de TIC's (Office, plataformas, etc.) & 1 & 10 & 7.14 & 2.427 \\
\hline \multirow{7}{*}{ Habilidades } & Capacidad de observación y análisis & 3 & 10 & 7.69 & 1.694 \\
\hline & Capacidad de síntesis & 3 & 9 & 6.83 & 1.599 \\
\hline & Trabajo en equipo & 2 & 10 & 7.46 & 2.160 \\
\hline & Expresión oral & 3 & 10 & 7.03 & 1.871 \\
\hline & Expresión escrita & 2 & 10 & 7.54 & 1.821 \\
\hline & Comprensión lectora & 2 & 10 & 7.69 & 1.843 \\
\hline & Creatividad & 3 & 10 & 7.97 & 1.706 \\
\hline \multirow{7}{*}{ Actitudes } & Emprendedurismo & 3 & 10 & 7.83 & 1.807 \\
\hline & Innovación & 4 & 10 & 7.77 & 1.750 \\
\hline & Liderazgo & 3 & 10 & 8.00 & 1.875 \\
\hline & Gestión & 3 & 10 & 7.57 & 1.803 \\
\hline & Reflexión y pensamiento crítico & 4 & 10 & 7.91 & 1.597 \\
\hline & Interés por temas económicos nacionales e internacionales & 5 & 10 & 8.33 & 1.562 \\
\hline & Responsabilidad & 4 & 10 & 8.46 & 1.559 \\
\hline
\end{tabular}

Fuente: Elaboración propia.

En términos generales, se observan promedios regulares respecto al perfil de ingreso de los estudiantes con respecto al perfil "ideal". La dimensión más favorecida fue la de Actitudes con un promedio de 7.98, mientras que las más desfavorecida fue la dimensión Conocimiento con 6.49 de promedio. La dimensión Habilidades obtuvo un promedio de 7. El promedio general con las tres dimensiones conjuntas es de 7.45 . 
DISEÑO DEL PERFIL DE INGRESO PARA LA INGENIERÍA EN GESTIÓN EMPRESARIAL DEL INSTITUTO SUPERIOR DE LIBRES.

Dentro de la dimensión Conocimientos, puede apreciarse que las características de Inglés básico y Conocimientos de Administración y Contabilidad básica, son las más bajas (también en comparación con las demás características de otras dimensiones). En este sentido, es importante retomar la perspectiva de Alvarado (2007), que señala que conociendo las fortalezas y debilidades de los estudiantes, es posible diseñar estrategias dirigidas a mejorar los aspectos en los que los estudiantes no cumplen adecuadamente en el marco del perfil de ingreso y puedan adquirir las características señaladas.

En la dimensión de Habilidades, Capacidad de síntesis y Expresión oral, son las características con menor valoración; generalmente, estas son características que con el paso del tiempo durante la estancia de los estudiantes en la institución se ven mejorando mediante diversas técnicas de enseñanza aprendizaje, además de que dentro del sistema del TECNM se realizan diversas presentaciones de proyectos de investigación y de inversión, donde los estudiantes aprenden a comunicar sus ideas y se desenvuelven adecuadamente ante una presentación pública. Además, como señalan Ysunza y De la Mora (2007), muchos de estos aspectos no se desarrollaron durante su paso en el nivel medio superior y durante su incorporación al nivel universitario esto puede mejorarse.

En la dimensión de Actitudes, Gestión e Innovación, son las características con menor valoración, pero se encuentran por encima del promedio general. En realidad, esto no resulta un problema grave, pues justamente estas son características que se potencializan durante el curso de la carrera mediante las asignaturas que se imparten.

\section{CONCLUSIONES}

Se ha observado, la diversidad de enfoques bajo las cuales se puede analizar la importancia de los perfiles de ingreso en los programas educativos de nivel superior, pero todos coinciden en que es importante definirlo adecuadamente para evitar problemas en las trayectorias escolares de los estudiantes y lograr buenos indicadores de eficiencia terminal, además de lograr que los alumnos adquieran todas las herramientas necesarias y puedan incorporarse al mercado laboral.

En el caso de los alumnos de la generación 2020 de la Ingeniería en Gestión Empresarial del ITSL, se pudo observar que se aproximan al perfil de ingreso diseñado. Si bien, la dimensión de Conocimientos fue la más baja, es un aspecto común en todos los programas educativos, sin MARTÍNEZ-ÁNGELES D. M., LOBATO-BÁEZ M., BELLO-RAMÍREZ M. I. 
DISEÑO DEL PERFIL DE INGRESO PARA LA INGENIERÍA EN GESTIÓN EMPRESARIAL DEL INSTITUTO SUPERIOR DE LIBRES.

duda, es un área de oportunidad porque se sabe con precisión las características más débiles que deben ser objeto de estrategias para mejorarlas como pueden ser cursos adicionales, asesorías tanto de parte de los profesores como de los mismos compañeros de clase, diseño de actividades teóricas y prácticas en las clases, entre otros.

Es importante señalar, que la pertinencia de un perfil de ingreso depende de muchos factores: tipo de universidad (pública o privada, autónoma o del sistema tecnológico), programa educativo (en función de las áreas del conocimiento), ubicación geográfica (zonas urbanas, rurales, industrializadas, etc.), principalmente. Por lo anterior, se debe poner mucha atención también en el perfil del estudiante, quizá ahí es donde ha habido confusión; una cosa es el perfil de ingreso y otra cosa es el perfil del estudiante de nuevo ingreso. Por eso, las aportaciones que se presentaron en la revisión bibliográfica, es diversa y pone atención en todos esos aspectos en conjunto.

Es posible proponer un único perfil de ingreso para esta ingeniería en todo el sistema TECNM, pues una de las bondades es que el sistema educativo es el mismo, los mapas curriculares también (a excepción de las especialidades, pues se diseñan con base en las características económicas y sociales de las zonas de influencia); en consecuencia, un diseño nacional de este perfil de ingreso permitiría mejorarlo continuamente y estandarizaría para los procesos de acreditación ante los organismos que correspondan.

\section{REFERENCIAS BIBLIOGRÁFICAS}

Alvarado, R. (2007). Perfil de ingreso ideal contra real de estudiantes de la Licenciatura en Gestión Turística, Facultad de Ciencias de la Administración, Campus IV; Universidad Autónoma de Chiapas. En Revista Iberoamericana de Producción Académica y Gestión Educativa, 4(8). https://www.pag.org.mx/index.php/PAG/article/view/700/942

De Garay, A. (2003). El perfil de los estudiantes de nuevo ingreso de las universidades tecnológicas en México. En El cotidiano, 9(122), 75-85. https://www.redalyc.org/articulo.oa?id=32512209\%253E\%2520ISSN\%25200186-1840

De Garay, A. (2003). Sujetos Itinerantes: Los Jóvenes Universitarios de la Universidad Autónoma Metropolitana. Tesis de Doctorado. UAM-Azcapotzalco. http://bindani.izt.uam.mx:3000/concern/tesiuams/cz30ps68g?locale=zh De Garay, A. y 
DISEÑO DEL PERFIL DE INGRESO PARA LA INGENIERÍA EN GESTIÓN EMPRESARIAL DEL INSTITUTO SUPERIOR DE LIBRES.

Sánchez, R. (2011). La modificación a la política de admisión para estudiar licenciatura en la UAM: Su impacto en el perfil de ingreso de los estudiantes a la Unidad Azcapotzalco. Un primer acercamiento. En Revista Mexicana de Investigación Educativa. 16(50), 885917. http://www.scielo.org.mx/pdf/rmie/v16n50/v16n50a10.pdf

Esparza, M. D. y López, R. (2011). Perfil de ingreso de alumnos con buen desempeño académico en el primer año de estudios. El caso de la Escuela de Diseño de la Universidad De La Salle Bajío. En Nova Scientia, 3(6), 95-120. http://www.redalyc.org/articulo.oa?id=203318388006

García, R. I. y López, C. A. (2011). Propuesta del perfil de ingreso y egreso del alumno para el bloque de administración de proyectos de la licenciatura en ciencias de la educación. En Revista El Buzón de Pacioli, Número Especial 74, 1-19 https://www.itson.mx/publicaciones/pacioli/Documents/no74/30.-_propue_1.pdf

Garibotti, F. y Ambroggio, G.A. (2012). Tomando perfil: ¿quién ingresa a Ciencias de la Educación? En Praxis Educativa (Arg) (1), 55-62. http://www.redalyc.org/articulo.oa?id=153124649006

González, A., Castro, E. y Bañuelos, D.D. (2011). Trayectorias escolares. El perfil de ingreso de los estudiantes de Ciencias Químicas: un primer abordaje para contrastación ulterior con otras disciplinas. En Revista Latinoamericana de Estudios Educativos, XLI, (3-4), 119-138. https://www.redalyc.org/articulo.oa?id=27022351006

González, M.S., Lara, A.M., Pineda, J. y Crespo, S. (2014). Perfil de ingreso de los alumnos de Enfermería, Facultades de Estudios Superiores Iztacala y Zaragoza, UNAM. En Enfermería Universitaria, 11(1), 11-18. http://www.scielo.org.mx/pdf/eu/v11n1/v11n1a3.pdf

Guerra, L., Guzmán, G. M. y Valdéz, G. del C. (2016). Ingeniería en Gestión Empresarial: un híbrido de los Institutos Tecnológicos. ANFEl. (4). https://anfei.mx/revista/index.php/revista/article/view/196/672\#: :text=Se\%20sabe\%20que \%20el\%20Programa,del\%202008\%20el\%20PA\%20mencionado.

Tecnológico Nacional de México (2020). Licenciaturas. https://www.tecnm.mx/?vista=Licenciaturas

Mireles, O. (2004). Integración de los jóvenes en el sistema universitario. Prácticas sociales, académicas y de consumo cultural. Por Adrián de Garay Sánchez. En Perfiles Educativos, 27(107), 161-165.

http://www.iisue.unam.mx/perfiles/articulo/2005-107-integracion-de-los-jovenes-en-el-sistemauniversitario-practicas-sociales-acadÉmicas-y-de-consumo-cultural-adrian-de-garaysanchez.pdf 
DISEÑO DEL PERFIL DE INGRESO PARA LA INGENIERÍA EN GESTIÓN EMPRESARIAL DEL INSTITUTO SUPERIOR DE LIBRES.

Rojas, L. (2014). Asociación de las carreras de Ciencias, Ciencias económicas y Ciencias Sociales con el perfil de ingreso de sus estudiantes. En Revista de Ciencias Sociales (Cr), I(143), 43-56. https://www.redalyc.org/articulo.oa?id=15333871004

Vivas, R. J., Yanez, S. G. y Pérez, J. S. (2018). Perfil de ingreso de los estudiantes a la Facultad de Ciencias Agrícolas en la Universidad Central del Ecuador. En Sophia, 14(2), 35-45. https://www.redalyc.org/articulo.oa?id=41375719400

Ysunza, M. y De la Mora, S. (2007). El perfil de ingreso del estudiante joven: una base para su incorporación al sistema universitario. En Memorias del IX Congreso Nacional de Investigación Educativa (CNIE) del Consejo Mexicano de Investigación Educativa A.C.

http://www.comie.org.mx/congreso/memoriaelectronica/v09/ponencias/at16/PRE11789086 21.pdf 